(C) 2013 IEEE. Personal use of this material is permitted. Permission from IEEE must be obtained for all other uses, in any current or future media, including reprinting/republishing this material for advertising or promotional purposes, creating new collective works, for resale or redistribution to servers or lists, or reuse of any copyrighted component of this work in other works. 


\title{
A Statistical Approach for Uncertain Stability Analysis of Mobile Robots
}

\author{
Mohammad Norouzi, Jaime Valls Miro and Gamini Dissanayake
}

\begin{abstract}
Stability prediction is an important concern for mobile robots operating in rough environments. Having the capacity to predict areas of instability means pro-actively being able to plan safer traversable paths. The most influential tip-over stability measures are based on two criteria, the robot's center of mass (CM) and the supporting polygon (SP) defined by the convex area spanned between the ground contact-points. However, there is significant uncertainty associated with many parameters in the planning pipe-line: the actual robot kino-dynamic model, its localisation in the ground, and the terrain models, particularly in uneven terrain. This article proposes a statistical analysis of stability prediction to account for some of the uncertainties. This is accomplished using the force angle (FA) stability measure for a reconfigurable multi-tracked vehicle fitted with flippers, a manipulator arm and a sensor head. Probability density function (PDF) of contact-points, CM and the FA stability measure are numerically estimated, with simulation results performed on the open dynamics engine (ODE) simulator based on uncertain parameters. Two techniques are presented: a conventional Monte Carlo scheme, and a structured unscented transform (UT) which results in significant improvement in computational efficiency. Experimental results on maps obtained from a range camera fitted on the sensor head while the robot traverses over a ramp and a series of steps are presented that confirms the validity of the proposed probabilistic stability prediction method.
\end{abstract}

\section{INTRODUCTION}

Mobile vehicles are frequently employed in unstructured environments where high levels of mobility are required. These systems have been performing a significant role in field environments missions such as search and rescue [1], agricultural vehicle, mobile manipulators [2] and planetary surface exploration [3]. In such missions, avoiding tip-over is a major concern because it often results in collateral damage to the robot and the general surrounding environment [4].

Stability prediction is an important task to successfully plan safe paths on challenging terrain. Robot's tip-over stability is a function of the robot configuration, environment geometry and the position of robot over the terrain. For an articulated robot as the iRobot Packbot shown in Fig. 1 in a mock-up Urban Search and Rescue (USAR) arena, moving on-board arm attached to the robot, significantly impacts the position of CM. Moreover, swinging the flippers (the two small front subtracks) can generally lead to changes in the SP of the robot, defined by the contact-points of the robot on the terrain.

Several tip-over stability measures have been proposed in the literature to evaluate the stability of a robot and predict

All authors are with the Faculty of Engineering and IT, University of Technology Sydney (UTS), Sydney NSW 2007, Australia. \{mohammad.norouzi, jaime.vallsmiro, gamini.dissanayake\} duts.edu.au

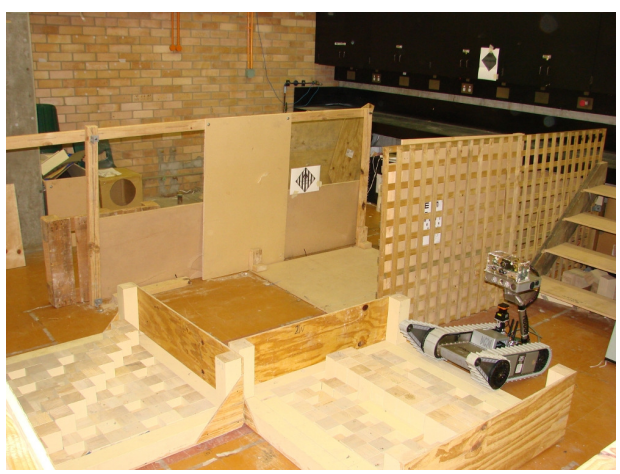

Fig. 1: The iRobot Packbot robot with additional sensor head on a mock-up USAR arena.

unstable conditions. The three most common stability metric algorithms were verified on the packbot tracked robot in [4], the same platform used in this paper for validation of the analysis hereby proposed. FA was proven to be one of the most effective stability measures, and is generally more widely used as it exhibits a more simplistic geometric interpretation [1]. An alternative real-time tip-over stability criteria for a reconfigurable tracked mobile platform on slopes was derived in [5] on the basis of load transfers by judging the supporting forces generated at the concerned tracked-terrain contact-points. The algorithm considered the contact-points to be fixed under the sprockets in order to describe the interactions between tracks and terrain. This is a strong assumption for the case of highly unstructured terrains, such as those featuring rubble, or for tracked robots in general, where contact-points can lay anywhere along the tracks, even more so if reconfigurability is also present (e.g. via flippers).

A study of the influence of a variable CM and the shape of the SP to plan safe traversable paths for the case of reconfigurable robots was also proposed in [1], where known local terrains and robot poses were assumed. Given uncertainties in the various parameters involved, a number of works have been developed in the literature. A motion planning algorithm for a wheeled mobile robot model that included some arbitrary fixed uncertainties in the terrain measurements and rover localisation was presented in [6]. A statistical method for planetary surface exploration focused their efforts on incorporating uncertainty in two deformable soil parameters, cohesion and internal friction angle is presented in [3]. The mobility prediction for the wheeled robot was performed via calculating wheelterrain interaction forces and vehicle dynamic motions. A 


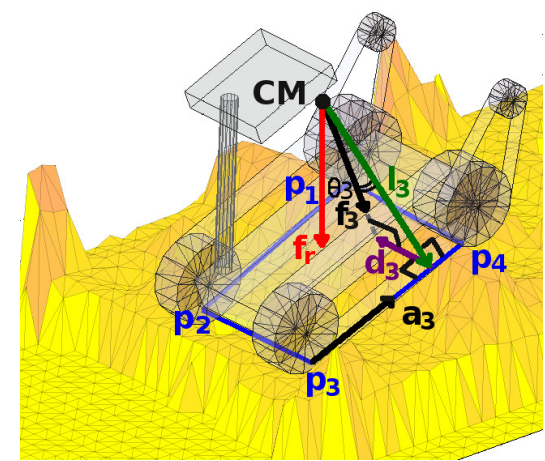

Fig. 2: 3D Force Angle stability for $i=3$ (CM's position has been shifted up and vectors scaled for easier visualisation).

stochastic response surface method (SRSM) was used as a functional approximation technique to obtain an equivalent system model with reduced complexity. The computational efficiency of SRSM was confirmed through comparison with a standard Monte Carlo (SMC) method [7], and the Latin hypercube sampling Monte Carlo method.

More recently, two polynomial chaos approaches for mobile robot dynamics prediction given uncertainty in obstacle's height, vehicle mass and surface type was presented in [8]. A ground vehicle simulator based on the ODE [9] was utilised to compare the results from the experiments conducted with a wheeled (P3-AT) mobile robot. The accuracy of these polynomial chaos approaches methods were validated through a baseline reference with the results obtained from the SMC approach. The robot was made to traverse over step obstacles and to compute the inclination of the robot, under the strong assumption that the wheels always remained in contact with the terrain, not necessarily the case for more unstructured environments, e.g. rubble, steps, etc.

The majority of the tip-over stability criteria mentioned above is predicated on the position of the CM and the SP. While there are propositions that take into account the uncertainties associated to some parameters, most rely on deterministic analysis that assume precise knowledge of the terrain physical model, robot pose and vehicle true configurations. Yet generally only sparse (often occluded) uncertain terrain model estimates can be drawn from sensors such as pointcloud cameras, vision or three-dimensional (3D) lasers while operating in realistic unstructured environments. Moreover, localisation in ruggedised 3D environments is significantly more challenging and uncertain than in well-known structured areas. Also, robot configuration may not be accurately known given controller residuals due to noisy feedback data from the robot joints and mechanical play. There is little research that explicitly addresses the challenges of robot stability analysis in uneven terrain while considering a wider range of the parametric uncertainties involved.

In this paper, a statistical framework for stability prediction using the FA criteria is presented that takes into account a realistic set of the uncertainties that can be expected to be present when planning in unstructured domains. Through an iterative dynamics simulation process, it will be shown how a probabilistic representation of the contact-points prediction and the stability metric can be derived. Simulation and experimental results of a multi-tracked robot (fitted with flippers, a manipulator arm and local (up to $5 \mathrm{~m}$ ) terrain data obtained from a ranging camera) while traversing over a ramp and a hill step-field are presented to validate the effectiveness of the proposed statistical approach.

\section{The FA Stability Metric}

Mobile robots usually move slowly over uneven terrains and a quasi-static stability analysis are shown to be appropriate for such scenarios [6]. Given location of contact-points and CM of robot in global coordinate frame, the FA stability measure can be computed as described in [2]. The $n$ out-most contact-points $\mathbf{p}_{\mathbf{i}}, i=\{1, \ldots, n\}$ are numbered in counterclockwise order as depicted in Fig. 2. The tip-over axes $\mathbf{a}_{\mathbf{i}}$ are defined as the segments that join the ground contact-points, and the set of the resulting convex polygon will be referred to as the SP. The vectors that intersect $\mathrm{CM}$ with each $\mathbf{a}_{\mathbf{i}}$ are referred to as $\mathbf{l}_{\mathbf{i}}$. Under quasi-static assumption, the net force $\mathbf{f}_{\mathbf{r}}$ acting on the system's CM will come from the gravitational loading term. Then for a given tip-over axis $\mathbf{a}_{\mathbf{i}}$, the components of $\mathbf{f}_{\mathbf{r}}$ which acts about the tip-over axis is calculated as $\mathbf{f}_{\mathbf{i}}$. The stability angle $\theta_{i}$ is computed as the angle between $\mathbf{f}_{\mathbf{i}}$ and the tip-over axis normal $\mathbf{l}_{\mathbf{i}}$. Critically, the FA stability measure is also sensitive to $\mathbf{d}_{\mathbf{i}}$, the minimum length vector from $\mathbf{a}_{\mathbf{i}}$ to $\mathbf{f}_{\mathbf{i}}$, hence defining the $i$ th stability measure $\beta_{i}$ as:

$$
\beta_{i}=\theta_{i}\left\|\mathbf{d}_{\mathbf{i}}\right\|\left\|\mathbf{f}_{\mathbf{i}}\right\|, \quad i=\{1, \ldots, n\}
$$

The example in Fig. 2 shows these derivations for $n=4$ and $i=3$. The overall robot's FA measure $\beta$ is defined as:

$$
\beta=\min \left(\beta_{i}\right), \quad i=\{1, \ldots, n\}
$$

\section{Contact-Points Prediction}

The robot-terrain prediction algorithm is based on the mathematical description of robot model in ODE, a widely used physical rigid body dynamics simulator, and a 3D model of the terrain obtained from the depth ranging information. The scheme is predicated on calculating the projection of the robot's geometric underside on the points defining the terrain underneath so as to derive the contact-points.

Under the assumption of quasi-static equilibrium, the influence of gravitational forces for a given robot pose and configuration can be calculated as an iterative process. To that end, the vehicle is first assumed to be sitting on a hypothetical plane with no pitch or roll at a given position and orientation in world coordinates. The 2D robot position $(r x, r y, y a w)$ in the global reference frame and the angle of the arm and flippers joints $\left(\phi_{A}, \phi_{F}\right)$ constitute the input variables. From these set of initial conditions, the ODE simulator can determine the final pose of the non-convex polygon defining the robot on the terrain $(r z$,roll, pitch), the location of the $\mathrm{CM}$, plus a list of contact-points between the robot and the terrain. The 


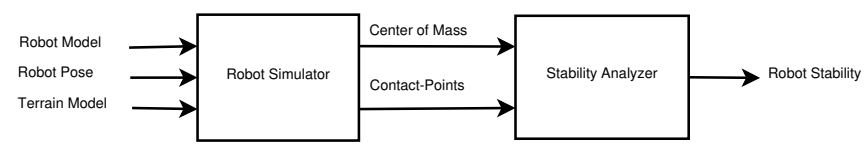

(a) Standard robot stability analyser.

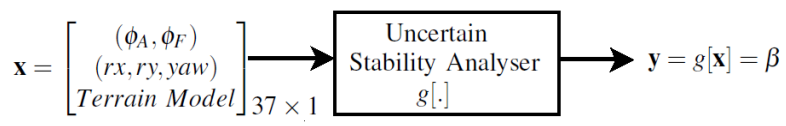

(b) Uncertain robot stability analyser.

Fig. 3: High-level pipe-line of the robot stability analyser. input: Robot model $\left(\phi_{A}, \phi_{F}\right)$, Robot Pose $(r x, r y, y a w)$ and meshed 3D Terrain Model.

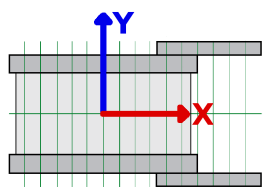

Fig. 4: The robot frame and sections $(2 \times 16)$ considered in this work in the stability analysis.

outermost points to form a convex polygon will become the contact-points needed to represent the SP of the vehicle when sitting on the terrain at that location. For more details on these derivations, the reader is referred to [1].

\section{The NeEd for a Probabilistic ApProACH}

A block diagram of the traditional robot stability analysis pipeline can be seen in Fig. 3a. If the input parameters can be assumed to be known deterministically, the stability criteria can be computed as described in Section II. In this work we argue there is a need to further introduce a mechanism to account for the uncertainty inherent in the key parameters that play a role in this analysis, e.g.:

1) Robot Configuration. Variations in $\phi_{A}$ and $\phi_{F}$, mainly due to mechanial wear-and-tear and encoder resolution, are assumed to be represented by a normal random distribution with variance of $3^{\circ}$, as indicated in the technical documentation of the robot.

2) Robot Pose. The noise associated with the robot's $2 \mathrm{D}$ pose is dependent on the accuracy of the localisation node. In this work, the study of stability analysis is restricted to the local terrain in front of the robot, up to $5 \mathrm{~m}$ in length. Hence, a conservative variance estimate of $1 \mathrm{~cm}$ per meter in $x$ and $y$ - linearly increasing with distance up to $5 \mathrm{~cm}$, and $2^{\circ}$ in orientation are assumed.

3) Terrain Model. The terrain, shown in Fig. 5 has been modelled by irregular triangulation ${ }^{1}$ of $3 \mathrm{D}$ point-cloud data obtained with an MS-Kinect [10] range camera. The model was built off-line by 3D ICP applied to the depth-image logs of the robot when manually navigating

\footnotetext{
${ }^{1}$ It should be noted that the proposed algorithm would work equally with regular triangulation meshes, height maps or other meshing techniques.
}

over the terrain at low speeds. The uncertainty values employed in this work are derived from [10], where it has been shown how the MS-Kinect sensor model error in the observed depth measurements increases quadratically with increasing distance, and reaches $4 \mathrm{~cm}$ at the maximum range of 5 meters.

The $z$ components of the vertices that describe the local section of the terrain where the robot may be located will be shifted up/down at the same time to analysed the effect of noise in the terrain model. For tracked vehicles such as the iRobot Packbot, contact-points are almost invariably located under the tracks. Hence, the proposition is for the robot to be divided into sections and the effect of variations in the terrain underneath be considered. Experiments were conducted with various sections until finer selections made little difference in the output of the contact-point predictions, settling for two sections in latitude, and 16 sections in the longitude direction as depicted by Fig. 4.

\section{UnCERTAINTY ANALYSIS METHOD}

To state the general problem, we have an n-dimensional vector random variable $\mathbf{x}$ with known mean $\hat{\mathbf{x}}$ and covariance $\mathbf{P}_{x x}$ and would like to predict the mean $\hat{\mathbf{y}}$ and covariance $\mathbf{P}_{y y}$ of a $\mathbf{m}$-dimensional vector random variable $\mathbf{y}$, where $\mathbf{y}$ is related to $\mathbf{x}$ by the non-linear transformation

$$
\mathbf{y}=g[\mathbf{x}]
$$

This is represented by Fig. $3 \mathrm{~b}$ in the context of the rescue robot problem presented in this paper, and described in more detail later in Section VII.

If $g[$.$] is a continuous and differentiable function, it is$ usually feasible to approximate it by a first-order Taylor series expansion about the point $\hat{\mathbf{x}}$, but when the transformation is highly non-linear or a deterministic analytical function doesn't exist, linear approximation methods are not valid and another statistical approaches should be employed. For instance a well known algorithm for estimating PDF of a general system's output response from a large set of repeated random inputs is the SMC method. The results of SMC will be more accurate as the size of input set increases, and the distribution of the pool is closer to the assumed pattern. This tendency to bigger set of inputs generally makes SMC computationally expensive, so other structured sampling techniques such as Latin hypercube sampling or importance sampling can be used to improve computational efficiency.

To speed up the transformation of means and covariances, [11] introduces UT filter to smaller set of input data. This technique suggests that rather than approximate the Taylor series to an arbitrary order, we can approximate the first three moments of the prior distribution accurately using a set of samples. Generally this method superficially resembles the SMC method, yet the samples are not drawn at random. Rather, the samples are deterministically chosen so that they capture specific information about the distribution. This filter is significantly easier to implement because it does 
not involve any linearisation steps, eliminating the derivation and evaluation of Jacobian matrices.

The $\mathrm{n}$-dimensional random variable $\mathbf{x}$ with mean $\hat{\mathbf{x}}$ and covariance $\mathbf{P}_{x x}$ is approximated by $2 n+1$ weighted samples or sigma points selected by the algorithm

$$
\begin{aligned}
X_{0} & =\hat{\mathbf{x}} \\
W_{0} & =k /(n+k) \\
X_{i} & =\hat{\mathbf{x}}+\left(\sqrt{(n+k) \mathbf{P}_{x x}}\right)_{i} \\
W_{i} & =1 /(2(n+k)) \\
X_{i+n} & =\hat{\mathbf{x}}-\left(\sqrt{(n+k) \mathbf{P}_{x x}}\right)_{i} \\
W_{i+n} & =1 /(2(n+k)) \\
i & =\{1, \ldots, n\}
\end{aligned}
$$

where $\left(\sqrt{(n+k) \mathbf{P}_{x x}}\right)_{i}$ is the $i$ th row or column of the matrix square root of $(n+k) \mathbf{P}_{x x}$ and $W_{i}$ is the weight that is associated with the $i$ th point. $k \in \mathbb{R}$, can be any number (positive or negative) providing that $(n+k) \neq 0$. For Gaussian distribution, a useful heuristic is to select $n+k=3$ to minimise the difference between the moments of the standard Gaussian and the sigma points up to the fourth order [11].

Given the set of samples generated by Eq. 4, the transformation of means and covariances procedure is as follows:

1) Each sigma point is instantiated through the process model to yield a set of transformed samples

$$
Y_{i}=g\left[X_{i}\right], \quad i=\{0, \ldots, 2 n\}
$$

2) The transferred mean is computed as

$$
\hat{\mathbf{y}}=\sum_{i=0}^{2 n} W_{i} Y_{i}
$$

3) The transferred covariance is computed as

$$
\mathbf{P}_{y y}=\sum_{i=0}^{2 n} W_{i}\left\{Y_{i}-\hat{\mathbf{y}}\right\} \times\left\{Y_{i}-\hat{\mathbf{y}}\right\}^{T}
$$

The mean and covariance are calculated using standard vector and matrix operations, which means that the algorithm is suitable for any choice of process model, and implementation is convenient because it is not necessary to evaluate the Jacobian matrixes, as in e.g. an Extended Kalman Filter.

\section{THE Robot MODEL}

The robot platform used in the simulations and experiments in this work is the multi-tracked iRobot Packbot, depicted in the USAR mock-up arena in Fig. 1. It consists of a skidsteer vehicle base, two small flippers in front of robot and a manipulator arm attached via a 1 Degree of Freedom (DoF) shoulder joint. It carries a 2-DoF pan-and-tilt unit equipped

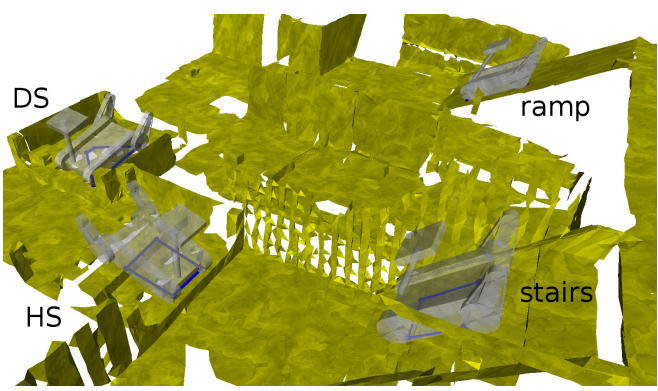

Fig. 5: Whole 3D USAR test arena and robot model at four locations (HS, DS, ramp and stairs).

with several sensors, lights and cameras including a MSKinect and an infrared camera. The robot's CM with respect to local frame is defined by:

$$
\mathbf{C M}=\frac{\sum_{j} \mathbf{p}_{\text {mass }_{j}} m_{j}}{m_{\text {tot }}}=\left[C M_{x} C M_{y} C M_{z}\right]^{T}
$$

where $m_{j}$ is the $j$ th lumped mass at location $\mathbf{p}_{\text {mass }}$ in the robot frame and $m_{t o t}$ is the total robot mass. As depicted in Fig. 4, $X$ is the robot's roll axis, $Y$ the pitch axis and $Z$ the direction normal to the platform, or yaw axis. The robot's coordinate frame origin is assumed to be located at the center of the support rectangular polygon formed when the robot's body is parallel to the horizontal plane. The weight of the base of robot and sensor head are $21.6 \mathrm{~kg}$ and $3.8 \mathrm{~kg}$ respectively. It is $69 \mathrm{~cm}$ long and $41 \mathrm{~cm}$ wide. The width of the main tracks is $7.5 \mathrm{~cm}$. These parameters, the geometries of the robot model and their kinematic constraints are described in the ODE with a set of fixed joints for non-moving parts, and two moving joints for the arm and the flippers.

\section{Simulation Results}

The simulation aims to estimate the distributions of contactpoints and stability measure $\beta$ under the assumption of a Gaussian model distribution of uncertainty in the robot configuration, environment geometry as well as the position of the robot over the terrain. The distribution of the contact-points is not really necessary for the uncertainty analysis, yet it is provided here to quantify the validity of the UT calculations. The result of SMC is compared with the more efficient structured sampling approach UT explained in Section V. The dimension of $\mathbf{x}$ in Eq. 4 is considered as $n=3+2+2 \times 16=37$, i.e. 3 DoF for the robot's 2D position (rx, ry, yaw), 2 for the robot's configuration $\left(\phi_{A}, \phi_{F}\right)$ and 16 for the number of sections in longitude direction as shown in Fig. 4. The robot was placed in four different terrain topologies including a ramp, stairs and two reconfigurable wooden cubic obstacles arranged in diagonal step-field (DS) and hill step-field (HS) arrangements. The 3D model of of USAR test arena and the four robot positions for this experiment is shown in Fig. 5.

In each position, the uncertainty analysis of contact-points and $\beta$ are first carried out with the well known SMC technique with 1000 iterations, while UT requires only 75 iterations. As depicted in the examples of Fig. 6 and Fig. 7, the "ideal" 


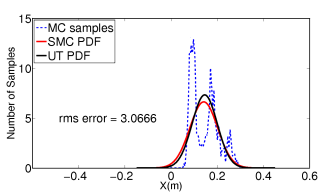

(a) cp1.x

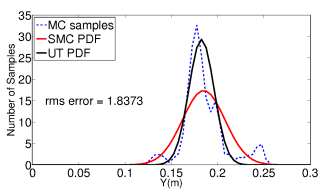

(e) cp1.y

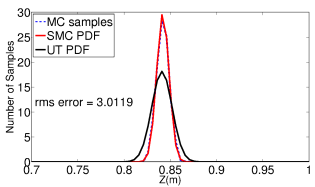

(i) $\mathrm{cp} 1 . \mathrm{z}$

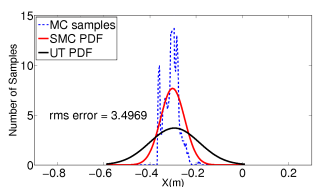

(b) cp2.x

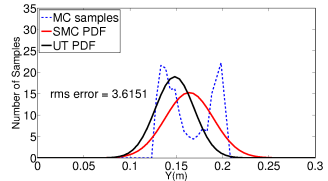

(f) cp2.y

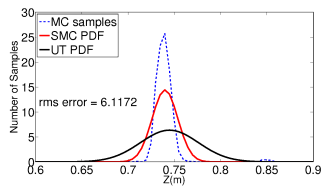

(j) cp2.z

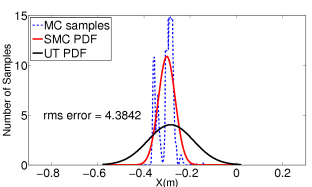

(c) cp3.x

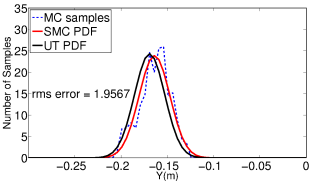

(g) cp3.y

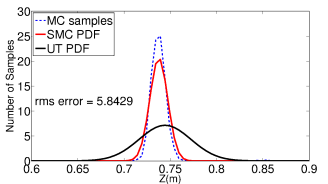

(k) cp3.z

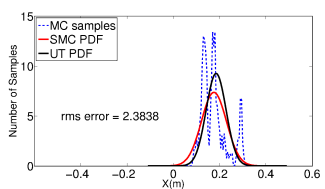

(d) cp4.x

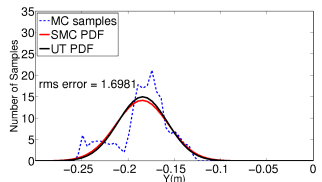

(h) cp4.y

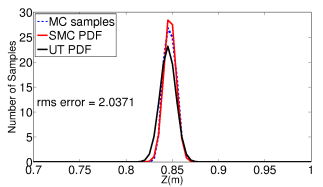

(1) cp4.z

Fig. 6: The distribution of contact-points over HS ( $x$ and $y$ in robot frame, $z$ in global frame), $(k=1)$.

\begin{tabular}{|c|c|c|c|c|c|c|c|}
\hline & cp1 & cp2 & cp3 & cp4 & CM & $\beta$ & mean \\
\hline HS & 2.639 & 4.410 & 4.061 & 2.040 & 1.888 & 1.127 & 2.694 \\
\hline DS & 2.342 & 3.826 & 3.148 & 2.056 & 1.763 & 2.331 & 2.577 \\
\hline ramp & 2.705 & 4.006 & 3.790 & 4.559 & 1.455 & 3.004 & 3.253 \\
\hline stairs & 1.582 & 2.464 & 4.475 & 3.828 & 0.710 & 2.600 & 2.610 \\
\hline
\end{tabular}

TABLE I: The rms errors(\%) between UT and Monte Carlo samples with $k=1$ (average $=2.7080 \%)$.

\begin{tabular}{|c|c|c|c|c|c|c|c|}
\hline & cp1 & cp2 & cp3 & cp4 & CM & $\beta$ & mean \\
\hline HS & 3.884 & 5.310 & 3.918 & 3.543 & 5.504 & 2.743 & 4.150 \\
\hline DS & 4.442 & 5.332 & 5.004 & 1.905 & 2.697 & 2.918 & 3.716 \\
\hline ramp & 3.296 & 3.084 & 4.901 & 5.284 & 3.518 & 3.013 & 3.849 \\
\hline stairs & 2.191 & 2.628 & 1.419 & 3.110 & 5.911 & 3.830 & 3.181 \\
\hline
\end{tabular}

TABLE II: The rms errors(\%) between UT and Monte Carlo samples with $k=-34$ (average $=3.7244 \%$ ).

PDF using the resulting SMC mean and covariance values are plotted in Red. The original SMC samples are plotted in dashed Blue around this PDF for comparison purpose.

The mean and covariance was then extracted using the UT method, with $k=3-n=-34$ as suggested in [11]. When $k$ is negative, it is possible that the predicted covariance will not be positive semi-definite. In such cases, the resulting PDFs don't follow closely the SMC samples, and trial-and-error is recommended to evaluate the covariance about the projected mean [11]. As suggested, simulations were repeated to find a more suitable value for $k$, with a positive value of $k=1$ significantly improving the rms error over a range of values.

Given space limitations, only distributions of contact-points for the HS test position and $k=1$ are depicted in Fig. 6. The PDF using covariance and mean obtained from UT are plotted in Black. The average rms error of each contact-point and $\beta$ in the four positions are summarised in Tables I and II for $k=1$ and $k=-34$ respectively. The PDFs of $\beta \mathrm{s}$ in the four positions are displayed in Fig. 7 for $k=1$.

\section{EXPERIMENTAL RESULTS}

To validate the results, the robot was made to traverse over the actual ramp and HS following a straight trajectory and constant reduced speed. A localiser using odometry and 2D range data from an auto-levelled laser scanner was employed to derive an estimate of the robot pose $(r x, r y, y a w)$ with a previously built 3D mesh of the arena, depicted in Fig. 5. As the platform has no suspension and the terrain is rigid, pitch and roll measurements from an on-board IMU can be assumed to be a fair reflection of the vehicle's attitude when sitting on the terrain. The robot's configuration $\left(\phi_{A}, \phi_{F}\right)$ was recorded from the actual on-board encoders during the experiments. The data from these tests was then analysed off-line to calculate the statistical properties of contact-points and stability measures.

The inclination of the ramp illustrated in Fig. 5 is $30^{\circ}$. The results of the ramp experiment are illustrated in Fig. 8a and Fig. 8c (roll is mostly kept constant around zero during the motion, hence not displayed as variations are insignificant). As shown in Fig. 8a, real inclination data is very close to that inferred by the simulator. The stability measure from a single simulation and mean value driven using UT in each point is depicted in Black and Red in Fig. 8c respectively. Also the standard deviation $\sigma(68 \%)$ and $2 \times \sigma(95 \%)$ around the mean are depicted in dashed Red and Blue. The measured $\beta$ and its mean value up to $\sigma$ is always positive which shows a convenient stability.

HS is an example to simulate common block obstacles, like rubble or unlevelled floors. The HS set-up illustrated in Fig. 5 is composed of three successive $10 \mathrm{~cm}$ steps, two traversed 


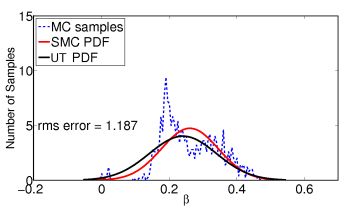

(a) $\mathrm{HS}$

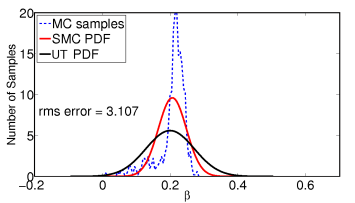

(c) ramp

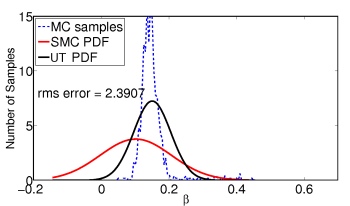

(b) DS

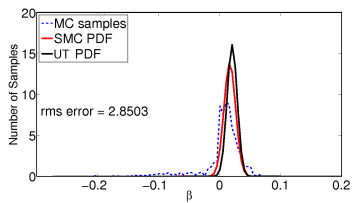

(d) stairs
Fig. 7: The distribution of $\beta \mathrm{s}$ in different positions $(k=1)$.

"up", and one "down". The results of the experiment over the HS are illustrated in Fig. 8b and Fig. 8d in the same way as was earlier depicted for the ramp (roll's variations are insignificant, thus not shown). As can be seen in Fig. 8b, the real inclination data is also closely captured by the simulator except at around $8 \mathrm{~s}$ and $17 \mathrm{~s}$, when the robot tipped-over and had to be manually handled and returned to the HS to prevent a fatal crash. Although the calculated mean value for $\beta$ can be seen to be just positive over the path at those instances, $\sigma$ uncertainty analysis shows the robot tipping-over at those instances (when the crossing over the steps takes place).

Comparing these two examples shows that despite of the smaller inclination in the HS configuration, in comparison with ramp, robot is still more stable over the ramp than HS. Assuming a fixed SP and calculating stability based on IMU data (like the approach in [4]) will lead to apparent stability, yet that is not the case. The traditional deterministic stability analysis method with variable SP can be regarded as fairly reliable over simple topologies like ramps, but can't predict instability over more challenging obstacles like HS where the uncertainty in the input parameters can have a significant influence on the output stability metric.

Another interesting observation can be seen in Fig. 7d, where on the stairs $\beta$ mean is only marginally stable, yet in the real experiments the robot was more often than not tipping over, vindicating once again the need to plan for the margins of the stability that this work advocates for.

\section{CONCLUSIONS}

Mobile robots are frequently employed in ruggedised environments where stability prediction is critical to the success of the mission. The most influential tip-over stability measures are based on two criteria, the CM and the SP. This paper argues that in uneven terrain, significant uncertainties are associated with the robot model, localisation and terrain parameters which need to be taken into account. A statistical method for stability prediction using the well known FA stability measure has been presented which can take into account these uncertainties.

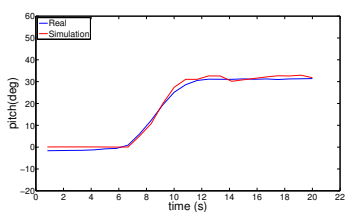

(a) pitch over ramp

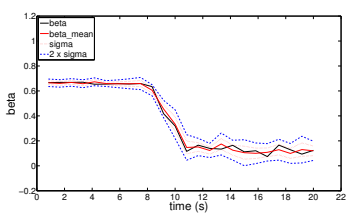

(c) $\beta$ over ramp

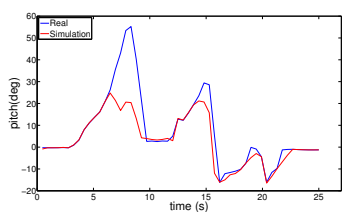

(b) pitch over HS

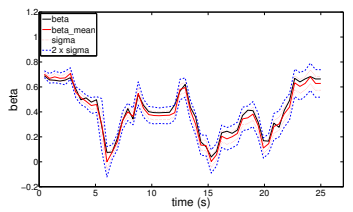

(d) $\beta$ over HS
Fig. 8: Experimental and simulation results over ramp and HS.

PDF of contact-points, CM and FA stability measure have been estimated, with simulation results from the uncertain parameters performed on the ODE. The proposed method has been validated with two techniques: a conventional standard Monte Carlo scheme, and a structured sampling approach which shows significant improvement in computational efficiency. Experimental results of a multi-tracked robot fitted with flippers and a manipulator arm travelling over a ramp and a hill step-field have been presenteded that confirm the utility of the proposed statistical stability prediction method.

\section{REFERENCES}

[1] M. Norouzi, J. V. Miro, and G. Dissanayake, "Planning high-visibility stable paths for reconfigurable robots on uneven terrain," in IEEE/RSJ International Conference on Intelligent Robots and Systems (IROS), Portugal, October 2012, pp. 2844-2849.

[2] E. Papadopoulos and D. A. Rey, "The force angle measure of tipover stability margin for mobile manipulatiors," Vehicle System Dynamics, vol. 33, no. 1, pp. 29-48, January 2000.

[3] G. Ishigami, G. Kewlani, and K. Iagnemma, "Predictable mobility," IEEE Robotics and Automation Magazine, vol. 16, no. 4, pp. 61-70, December 2009.

[4] P. R. Roan, A. Burmeister, A. Rahimi, K. Holz, and D. Hooper, "Realworld validation of three tipover algorithms for mobile robots," in Proc. IEEE International Conference on Robotics and Automation (ICRA), Anchorage, Alaska, USA, May 2010, pp. 4431-4436.

[5] Y. Liu and G. Liu, "Interaction analysis and online tip-over avoidance for a reconfigurable tracked mobile modular manipulator negotiating slopes," IEEE/ASME Transactions on Mechatronics, vol. 15, no. 4, pp. 623-635, August 2010.

[6] K. Iagnemma and S. Dubowsky, Mobile Robot in Rough Terrain. Berlin, Germany: Springer Tracts in Advanced Robotics, 2004, vol. 12.

[7] Rubinstein and R. Y, Simulation and the Monte Carlo method. New York: Wiley, 1981.

[8] G. Kewlania, J. Crawfordb, and K. Iagnemma, "A polynomial chaos approach to the analysis of vehicle dynamics under uncertainty," Vehicle System Dynamics, vol. 50, no. 5, pp. 749-774, May 2012.

[9] R. Smith, Open Dynamics Engine, 2005. [Online]. Available: http://www.ode.org/

[10] K. Khoshelham and S. O. Elberink, "Accuracy and resolution of kinect depth data for indoor mapping applications," Journal of Sensors, vol. 12, no. 1, pp. 1437-1454, January 2012.

[11] S. Julier, J. Uhlmann, and H. F. Durrant-Whyte, "A new method for the nonlinear transformation of means and covariances in filters and estimators," IEEE Transactions on Automatic Control, vol. 45, no. 3, pp. 477-482, March 2000. 